Author has nothing to disclose with regard to commercial support.

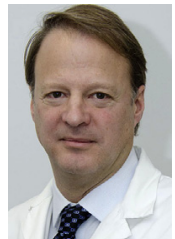

\section{REPLY: SHOULD WE "DOUBLE BREAST" THE SINO-TUBULAR JUNCTION WITH AN ADVENTITIAL FLAP DURING ROOT REPAIR IN TYPE A DISSECTION?}

\section{Reply to the Editor:}

Motekallemi and colleagues report an interesting modification to the aortic root repair technique in type A dissection proposed by Yang and colleagues ${ }^{1}$ in a recent issue of the Journal. Yang and colleagues stressed the importance of using a 5-0 PROLENE running stitch to reapproximate the dissected layers of the aortic wall without use of glue or Teflon felt and ensuring graft-to-intima apposition by telescoping the Dacron graft within the root. They reported excellent mid-term outcomes with no root pseudoaneurysm. To increase strength on the proximal anastomosis and minimize the impact of needle holes, Motekallemi and colleagues propose to invaginate a layer of adventitia $(1 \mathrm{~cm})$ within the aortic root while approximating the dissected aortic layers with a running 5-0 PROLENE stitch.

Although different techniques are acceptable to solve a surgical problem, the principles of repair should rely on the objectives one wishes to accomplish. Regarding root repair during type A dissection, 3 principles should be respected: (1) restore aortic valve competency through commissure resuspension; (2) closure of the false lumen and avoiding residual or iatrogenic intimal tears to minimize late pseudoaneurysm formation; and (3) perform a hemostatic proximal anastomosis between the Dacron graft and the "neo-sinotubular" junction. Valve competency is restored by repositioning the commissure height but also by respecting the 3-dimensional valve geometry throughout the root repair. Residual intimal tear either through tears related to the dissection process or induced by the suturing technique are responsible in maintaining flow in the false lumen with the risk of late pseudoaneurysm. Although extensive tears in the root mandate a root replacement, small tears extending in the sinuses may be repaired primarily using small needles and incorporating the full thickness of the aortic wall. The use of a small layer of glue to reinforce closure of the dissected layers has been reported with success, albeit late complication of tissue necrosis has been recognized. ${ }^{2}$ In preparation for the proximal anastomosis, the dissected layers may be reapproximated with a running circumferential stitch.

I agree with Motekallemi and colleagues that root geometry is better preserved with a running suture as opposed to a horizontal stitch. Furthermore, occasional "locking" of the running suture may minimize the purse string effect of the running stitch. In addition to forcing the closure of the false lumen, the running stitch restores the upper 3-dimensional valve geometry mainly by restoring the appropriate intercommissural distance at the sino-tubular level and facilitates the subsequent proximal anastomosis. Invagination of the adventitia, as proposed in the present comment, reinforces the sino-tubular junction. However, as visualized in their figure, the ridge should be minimal and avoid excessive interposition of periadventitial fat. As with Teflon, undue exposure of adventitia within the lumen of the root may be thrombogenic and should be avoided. Selection of the graft diameter for the proximal anastomosis should be slightly smaller than the neo-sinotubular junction diameter to facilitate telescoping the graft within the root. Marking the position of the $3 \mathrm{com}-$ missures on the Dacron graft may facilitate respecting the intercommissural distance, hence preserving root geometry.

Yang and Motekallemi have outlined the importance of using small needles to minimize intimal tears and needle holes. In addition to small needles, basic surgical principles such as respecting the needle curvature, avoiding needle "prying," and excessive stitch traction should be respected to minimize iatrogenic tears while performing a secure proximal anastomosis in a type A dissection setting. Use of novel HEMO-SEAL PROLENE stitches (Johnson and Johnson, New Brunswick, NJ) with a one-to-one ratio between the needle and the PROLENE stitch may further decrease needle holes and iatrogenic intimal tears. Once again apposing the graft to the intima within the root increases hemostasis and minimizes exposure of thrombogenic tissue such as adventitia or Teflon.

In conclusion, Yang and Motekallemi report similar techniques to repair the aortic root in the setting of type A dissection. Meticulous use of small needles, proper valve resuspension, and false lumen obliteration with a running stitch at the neo sino-tubular ridge while preserving the root geometry are of paramount importance. Strengthening the sino-tubular ridge with an adventitial flap as described by Motekallemi and colleagues remains an option but should respect principles outlined for a secure aortic root repair in type A dissection.

Francois Dagenais, $M D$

Department of Cardiac Surgery

Institut Universitaire de Cardiology et Pneumologie de Québec

Québec, Québec, Canada

\footnotetext{
References

1. Yang B, Malik A, Waidley V, Kleeman KC, Wu X, Norton EL, et al. Short-term outcomes of a simple and effective approach to aortic root and arch repair in acute type A aortic dissection. J Thorac Cardiovasc Surg. 2018;155:1360-70.

2. Bhamidipati C, Cosellei JS, LeMaire SA. BioGlue in 2011: what is its role in cardiac surgery. J Extra Corpor Technol. 2012;44:6-12.
}

https://doi.org/10.1016/j.jtcvs.2019.07.033 